Bull. Austral. Math. Soc.

VOL. 48 (1993) [347-350]

\title{
THE CENTRE AND THE DEPTH OF THE CENTRE OF A TREE MAP
}

\section{XIANGDONG YE}

Let $X$ be a tree and $f$ be a continuous map from $X$ into itself. Denote by $P(f)$ and $R(f)$ the set of periodic points and the set of recurrent points of $f$ respectively. We show in this note that the centre is $\overline{P(f)}$ and the depth of the centre is at most 3. Furthermore we have $\overline{P(f)}=\overline{R(f)}$.

\section{INTRODUCTION}

Let $X$ be a compact metric space and $f \in C(X, X)$. A point $x \in X$ is said to be a nonwandering point of $f$ if for any neighbourhood $V_{x}$ of $x$ there exists $n \in \mathbf{N}$ such that $f^{n}\left(V_{x}\right) \cap V_{x} \neq \emptyset$. The set of nonwandering points of $f$ will be denoted by $\Omega(f)$. Let $\Omega_{1}(f)=\Omega(f), \Omega_{n}(f)=\Omega\left(f \mid \Omega_{n-1}\right), n \geqslant 2$. Then $\Omega_{\infty}(f)=\bigcap_{1}^{\infty} \Omega_{n}(f)$ is called the centre of $f$. The minimal $n \in \mathrm{N} \cup\{\infty\}$ such that $\Omega_{n}(f)=\Omega_{\infty}(f)$ will be called the depth of the centre of $f$.

We say that $x \in X$ is a periodic point of $f$ of period $n$ if there exists $n \in \mathbf{N}$ such that $f^{n}(x)=x$ but $f^{i}(x) \neq x, 1 \leqslant i \leqslant n-1$. If $n=1$ then $x$ will be called a fixed point of $f$. A point $x \in X$ is a recurrent point of $f$ if for every neighbourhood $V_{x}$ of $\boldsymbol{x}$ there is $n \in \mathbf{N}$ such that $f^{n}(x) \in V_{x}$. The sets of fixed points, periodic points and recurrent points of $f$ will be denoted by $F(f), P(f)$ and $R(f)$ respectively.

The notion of periodic point, recurrent point and centre of a continuous map $f$ from a compact metric space into itself is one of the most important notions in dynamical systems. In general it is difficult to determine the centre and the depth of the centre of $f$ and it is not always true that $\overline{P(f)}=\overline{R(f)}$. For example, let $f$ be an irrational rotation from a circle into itself. Then we have that $P(f)=\emptyset$ and $R(f)$ is the circle.

If $X=[0,1]$ it is known that $\Omega_{2}(f)=\overline{P(f)}$ and the centre is $\overline{P(f)}$ and the depth of the centre is at most 2 [1] (for a simple proof see [3]). In [2] this result is extended to continuous maps from $n$-od to itself having zero as a fixed point, where $n$-od $=\left\{z \in \mathbb{C}: z^{n} \in[0,1]\right\}$. In this note we shall generalise these results to general continuous tree maps. To state the results we start with some more notion.

By a tree we mean a compact connected one-dimensional branched manifold without cycles. A point $x \in X$ is a branched point of $f$ if there is a neighbourhood of $x$

Received 24 November 1992

Copyright Clearance Centre, Inc. Serial-fee code: 0004-9729/93 \$A2.00+0.00. 
which is homeomorphic to $\left\{z \in \mathbf{C}: z^{k} \in[0,1]\right\}$ for some $k \geqslant 3$. The set of branched points of $X$ is a finite subset of $X$ and will be denoted by $O=\left\{o_{1}, o_{2}, \ldots, o_{n}\right\}$. For $x \in X \backslash O$, there is a neighbourhood $V_{x}$ of $x$ such that $V_{x}$ is homeomorphic to $(0,1)$ or $(0,1]$. In the latter case we say that $x$ is an end point of $X$. We assume that the set of end points of $X$ is the finite set $\left\{e_{1}, e_{2}, \ldots, e_{t}\right\} \subset X$.

Let $\rho$ be a metric on a tree $X$. If $X_{1}, X_{2}$ are two subsets of $X$ then we define $\rho\left(X_{1}, X_{2}\right)=\inf \left\{\rho\left(x_{1}, x_{2}\right): x_{i} \in X_{i}, i=1,2\right\}$. Furthermore we assume that $\rho\left(\emptyset, X_{1}\right)>0$ for every subset $X_{1}$ of $X$.

Now we are ready to state the main results of this note.

Theorem 2.6. Let $X$ be a tree and $f \in C(X, X)$. Then $\overline{R(f)}=\overline{P(f)}$.

TheOREM 2.7. Let $X$ be a tree, $f \in C(X, X)$ and $O$ be the set of branched points of $X$. Then $\Omega_{2}(f)=\overline{P(f)} \cup O^{\prime}$, where $O^{\prime} \subset O$ and $\rho\left(O^{\prime}, \overline{P(f)}\right)>0$. Hence the depth of the centre is at most 3 and the centre is $\overline{P(f)}$.

\section{ThE PROOFS}

Through out this section we assume that $X$ is a tree and $f \in C(X, X)$. It is well known that $F(f) \neq \emptyset$. In the following we give a partial order on the tree $X$ which is useful in dealing with continuous tree maps.

Definition 2.1: Let $X$ be a tree. Then for every pair $x, y \in X,[x ; y]$ will be the unique segment connecting $x$ and $y$.

Definition 2.2: Let $e$ be an end point of the tree $X$. A partial order $<_{e}$ on $X$ is defined by: $x<_{e} y$ if and only if $x \in[e: y]$ and $x \neq y$.

Definition 2.3: Let $e$ be an end point of the tree $X$ and $X \backslash O=\bigcap_{1}^{n_{0}} I_{j}$. Define $<_{r}=<_{r\left(j_{0}, e\right)}$ on $X$ by: If $\bar{I}_{j_{0}}=\left[y_{1} ; y_{2}\right], y_{1}<_{e} y_{2}$ and $X_{2}=\left\{x \in X: y_{2}<_{e} x\right\} \cup\left[y_{1} ; y_{2}\right]$, $X_{1}=\left(X \backslash X_{2}\right) \cup\left[y_{1} ; y_{2}\right]$ then $z_{1}<_{r} z_{2}$ if and only if either $z_{1} \in X_{1} \backslash\left[y_{1} ; y_{2}\right], z_{2} \in X_{2}$ or $z_{2}<_{y_{2}} z_{1}$ if $z_{1}, z_{2} \in X_{1} ; z_{1}<_{y_{1}} z_{2}$ if $z_{1}, z_{2} \in X_{2}$ (we consider $X_{1}, X_{2}$ to be two subtrees of $X$ ).

Lemma 2.4. Let $X$ be a tree, $f \in C(X, X)$ and $X \backslash O=\bigcap_{1}^{n_{0}} I_{j}$. If $x<_{r} y \in$ $\left[y_{1} ; y_{2}\right]=I_{j_{0}}$ with $x<_{r} f(x), f(y)<_{r} y$ then $F(f) \cap[x ; y] \neq \emptyset$.

ProOF: Because $f([x ; y])$ is a connected subset of $X$ we know that there exist $x_{1}<_{r} y_{1} \in[x ; y]$ such that $x_{1}<_{r} f\left(x_{1}\right), f\left(y_{1}\right)<_{r} y_{1}$ and $f\left(x_{1}\right), f\left(y_{1}\right) \in I_{j 0}$. By the continuity of $f$ we can find $x_{2}<_{r} y_{2} \in\left[x_{1} ; y_{1}\right]$ such that $x_{2}<_{r} f\left(x_{2}\right), f\left(y_{2}\right)<_{r} y_{2}$ and $f\left(\left[x_{2} ; y_{2}\right]\right) \subset I_{j 0}$. It is well known that $F(f) \cap\left[x_{2} ; y_{2}\right] \neq \emptyset[1]$.

Lемма 2.5. Let $X$ be a tree and $f \in C(X, X)$. If $f([x ; y]) \supset[x ; y]$ for some $x, y \in X$ and $[x ; y] \cap O=\emptyset$ then $F(f) \cap[x ; y] \neq \emptyset$. 
Proof: From $f([x ; y]) \supset[x ; y]$ we know that there exist $x_{1}, y_{1} \in[x ; y]$ such that $f\left(x_{1}\right)=x, f\left(y_{1}\right)=y$. By the connectedness of $f\left(\left[x_{1}, y_{1}\right]\right)$ we can choose $x_{2}, y_{2} \in$ $\left[x_{1} ; y_{1}\right]$ such that $f\left(\left[x_{2} ; y_{2}\right]\right) \subset\left[x_{1}, y_{1}\right]$. So we have reduced our case to the interval case. The lemma follows from [1].

THEOREM 2.6. Let $X$ be a tree and $f \in C(X, X)$. Then $\overline{P(f)}=\overline{R(f)}$.

Proof: Let $x \in X \backslash \overline{P(f)}$ and $V_{x}$ be the open connected component of $X \backslash \overline{P(f)}$ such that $x \in V_{x}$.

Suppose $X \backslash O=\bigcap_{1}^{n_{0}} I_{j}$

We claim that $\left(V_{x} \cap I_{j_{0}}\right) \cap R(f)=\emptyset, \forall 1 \leqslant j_{0} \leqslant n_{0}$.

If it is not the case let $y \in\left(V_{x} \cap I_{j_{0}}\right) \cap R(f)$. Then there exists $n_{1} \in \mathbf{N}$ such that $f^{n_{1}}(y) \in V_{x} \cap I_{j_{0}}$. Let $g=f^{n_{1}}$. Without loss of generality we assume $y<_{e} g(y)$ for some end point $e$.

Suppose that $V_{x} \cap I_{j_{0}}=[a ; b], a<_{e} b$. We have $a<_{r} y<_{r} g(y)<_{r} b$. It follows that $z<_{r} g(z), \forall z \in[a ; b]$. Otherwise if there exists $y_{1} \in V_{z} \cap I_{j_{0}}$ such that $g\left(y_{1}\right)>_{r} y_{1}$ then $\left[y_{1} ; y\right] \subset f\left(\left[y_{1} ; y\right]\right)$ if $y_{1}<_{r} y$ or it is the case of Lemma 2.4. By Lemma 2.4 and 2.5 it is a contradiction.

Now suppose $z<_{r} g^{i}(z), \forall z \in[a ; b], 1 \leqslant i \leqslant n$. It follows that $y<_{r} g(y)<_{r}$ $g^{i+1}(y), 1 \leqslant i \leqslant n$. By the same reasoning $z<_{r} g^{n+1}(z), \forall z \in[a ; b]$. So we conclude that $z<_{r} g^{m}(z), \forall z \in[a ; b]$ and $m \in \mathrm{N}$. Hence $y<_{r} g(y)<_{r} g^{m}(y) \forall m \in \mathrm{N}$. That is to say $y \notin R(g)=R(f)$.

From $\left(V_{x} \cap I_{j_{0}}\right) \cap R(f)=\emptyset . \forall I_{j_{0}} \cap V_{x} \neq \emptyset$ we get $V_{x} \cap R(f)=\emptyset$ because if there is $i_{0} \in \mathbf{N}$ such that $o_{i_{0}} \in V_{x}$ then $o_{i_{0}} \notin R(f)$ (an isolated recurrent point is a periodic point).

To sum up $\overline{P(f)}=\overline{R(f)}$.

THEOREM 2.7. Let $X$ be a tree and $f \in C(X, X)$. Then $\Omega_{2}(f)=\overline{P(f)} \cup O^{\prime}$ where $O^{\prime} \subset O$ and $\rho\left(O^{\prime}, \overline{P(f)}\right)>0$. Hence the depth of the centre is at most 3 and the centre is $\overline{P(f)}$.

For this purpose we show the following lemma

Lemma 2.8. Let $f \in C(X, X)$ for a tree $X$ and $X \backslash O=\bigcup_{1}^{n_{0}} I_{j}$. If an open interval $J \subset I_{j_{0}}$ for some $1 \leqslant j_{0} \leqslant n_{0}$ satisfies $J \cap P(f)=\emptyset$, then $J \cap f^{n}(J \cap \Omega(f))=$ $\emptyset, \forall n \in N$.

Proof: If it is not the case then there exists $x \in J \cap \Omega(f)$ and $n \in \mathbf{N}$ such that $f^{n}(x) \in J, f^{i}(x) \notin J, 1 \leqslant i<n$. It is easy to see that $f^{i}(x) \neq f^{j}(x)$ if $0 \leqslant i<j \leqslant n$.

Without loss of generality we assume that $x<_{r} f^{n}(x)$. Choose the neighbourhood $V_{i}$ of $f^{i}(x)$ for $0 \leqslant i \leqslant n$ such that $V_{i} \cap V_{j}=\emptyset$ for $0 \leqslant i<j \leqslant n$ and $V_{n} \subset J$. Put 
$V=\bigcap_{0}^{n} f^{-i}\left(V_{i}\right)$, then $V$ is a neighbourhood of $x$. Since $x \in \Omega(f)$, there exists $l>0$ such that $V \cap f^{l}(V) \neq \emptyset$. Obviously $l>n$. As $f^{n}(V) \subset V_{n}$ and $f^{l-n}\left(V_{n}\right) \cap V \neq \emptyset$, there is $y \in V_{n}$ such that $f^{l-n}(y) \in V$. Hence $f^{l-n}(y)<_{r} y$. By the proof of Theorem 2.6, $z<_{r} f^{n m_{1}}(z), f^{m_{2}(l-n)}(z)<_{r} z, \forall z \in J, \forall m_{1}, m_{2} \in \mathrm{N}$. It is a contradiction if we choose $m_{1}=l-n, m_{2}=n$.

Proof of Theorem 2.7: Clearly $\overline{P(f)} \subset \Omega_{2}(f)$.

Let $C$ be a connected component of $X \backslash \overline{P(f)}$ and $X \backslash O=\bigcup_{1}^{n_{0}} I_{j}$. By the above Lemma 2.8 for every nonnegative integer $n$ we have $\left(C \cap I_{j}\right) \cap f^{n}\left(C \cap I_{j} \cap \Omega(f)\right)=\emptyset$ for all $1 \leqslant j \leqslant n_{0}$. Hence $C \cap I_{j} \cap \Omega\left(\left.f\right|_{\Omega(f)}\right)=\emptyset$. To sum up we have proved that $\Omega(f \mid \Omega(f)) \subset \overline{P(f)} \cup O^{\prime}$, where $O^{\prime} \subset O$ and $\forall x \in O^{\prime}, x$ is an isolated point of $\Omega_{2}(f)$ and $x \notin \overline{P(f)}$. So $\rho\left(O^{\prime}, \overline{P(f)}\right)>0$ and $\Omega_{3}(f)=\overline{P(f)}$.

\section{REFERENCES}

[1] Z. Nitecki, 'Topological dynamics on the interval', Progr. Math. 21 (Birkhauser, Boston), pp. 1-72.

[2] H. Wu, 'The center of continuous mapping on n-od'. Preprint, SISSA 162M (1990).

[3] J.C. Xiong, Kexue Tongbao 28 (1983), 21-23.

Department of Mathematics

USTC

Hefei

Anhui 230026

People's Republic of China 